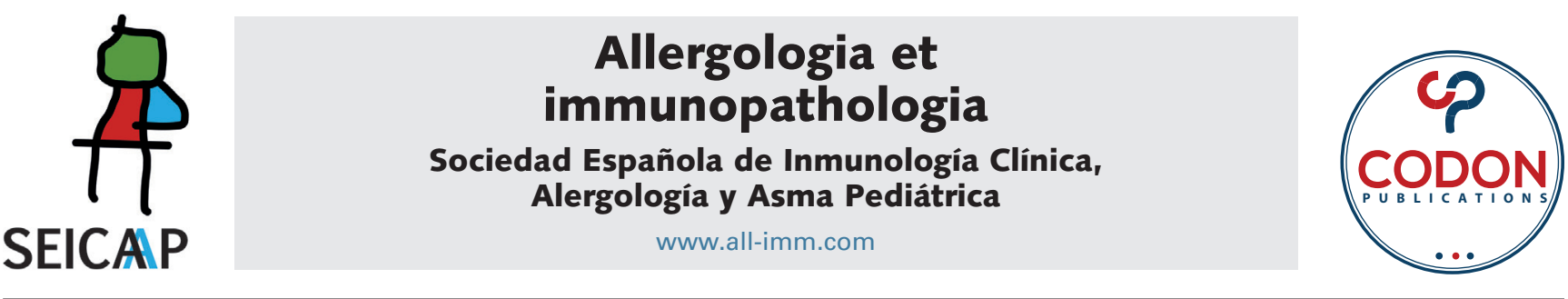

Original Article

OPEN ACCESS (c) $\underset{\mathrm{BY}}{\mathrm{NC}(\mathrm{FA}}$

\title{
MiR-216a-5p alleviates LPS-induced inflammation in the human bronchial epithelial cell by inhibition of TGF- $\beta 1$ signaling via down-regulating TGFBR2
}

\author{
Shan Liu ${ }^{a}$, Jianjun Li ${ }^{b}$ Liya Hu** \\ ${ }^{a}$ Department of Pediatrics, Zhejiang Quhua Hospital, Quhua City, Zhejiang Province, 324004, China \\ ${ }^{b}$ Department of Pediatrics, Lishui Peoples Hospital, Lishui City, Zhejiang Province, 323000, China
}

Received 2 June 2021; Accepted 29 July 2021

Available online 1 September 2021

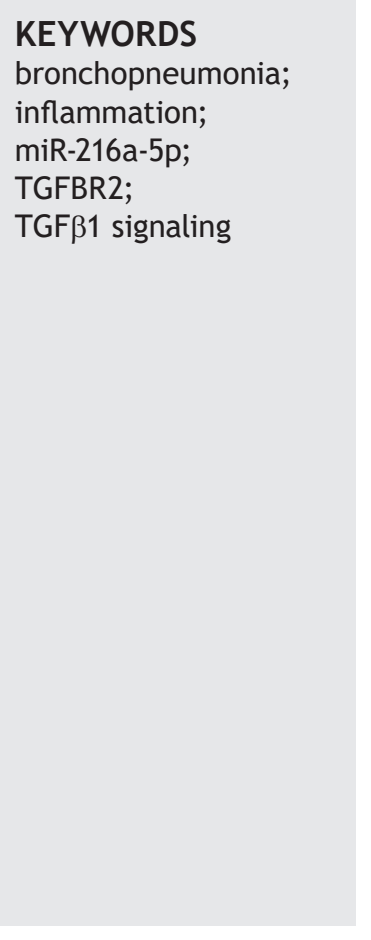

\begin{abstract}
Objective: Bronchopneumonia is a common respiratory infection disease and is the leading cause of hospitalization in children under 5 years of age. Inflammation is the primary response caused by bronchopneumonia. But the detailed underlying mechanism of inflammation in bronchopneumonia remains unclear. Therefore, this study focused on studying the effect of miR-216a-5p on inflammation induced by bronchopneumonia and investigate the potential mechanism underlying it.

Methods: Human bronchial epithelial cells (BEAS-2B) were stimulated using lipopolysaccharides (LPS) to trigger bronchopneumonia in vitro. The production of interleukin (IL)-1 $\beta$, IL-6, and Tumor necrosis factor (TNF)- $\alpha$ was measured using the enzyme-linked immunosorbent assay. The luciferase assay was conducted to explore the relationship between miR-216a-5p and TGFBR2. Quantitative real-time polymerase chain reaction and western blot were used to detect the gene expression.

Results: miR-216a-5p gene expression decreased in BEAS-2B cells stimulated by LPS. Overexpression of miR-216a-5p suppressed the elevated levels of IL-1 $\beta$, IL-6, and TNF- $\alpha$ induced by LPS. Transforming growth factor-beta receptor 2 (TGFBR2) proved to be a direct target of miR-216a-5p, and they negatively modulated TGFBR2 expression. In addition, overexpression of miR-216a-5p inhibited LPS-induced protein levels of TGFBR2,transforming growth factor (TGF)- $\beta 1$, and phosphorylation of SMAD family member 2 (smad2),. This ectopic expression of miR-216a-5p was restored by overexpressed TGFBR2.

Conclusion: miR-216a-5p was decreased in LPS-stimulated BEAS-2B cells. Overexpressed miR216a-5p suppressed LPS-induced inflammation in BEAS-2B cells by inhibition of TGF- $\beta 1$ signaling via down-regulating TGFBR2. miR-216a-5p may be a valuable target for anti-inflammation treatment in bronchopneumonia.
\end{abstract}

*Corresponding author: Liya Hu, Department of Pediatrics, Lishui Peoples Hospital, Lishui, Zhejiang Province, 323000, China. Email address: huliya_666@163.com 
Bronchopneumonia is a common respiratory infection disease and is the main cause of hospitalization in children under 5 years of age. Inflammation is a primary response caused by bronchopneumonia. But the detailed underlying mechanism of inflammation in bronchopneumonia remains unclear. Therefore, this study focused on studying the effect of miR-216a-5p on inflammation caused by bronchopneumonia and investigate the potential mechanism underlying it. In this study, human bronchial epithelial cells (BEAS-2B) were stimulated using lipopolysaccharides (LPS) to trigger bronchopneumonia in vitro. miR-216a-5p was decreased in BEAS-2B cells stimulated by LPS. Overexpression of miR-216a-5p suppressed the elevated levels of interleukin (IL)-1 $\beta$, IL-6, and Tumor necrosis factor (TNF)- $\alpha$ induced by LPS. Transforming growth factor-beta receptor 2 (TGFBR2) proved to be a direct target of miR-216a-5p, and they negatively modulated TGFBR2 expression. In addition, overexpression of miR-216a-5p inhibited LPS-induced protein levels of TGFBR2,transforming growth factor-beta 1 (TGF- $\beta 1$ ), and phosphorylation of SMAD family member 2 (smad2. This ectopic overexpression of miR-216a-5p was restored by overexpressed TGFBR2. In conclusion, miR-216a-5p was decreased in LPSstimulated BEAS-2B cells. Overexpressed miR-216a-5p suppressed LPS-induced inflammation in BEAS-2B cells by inhibition of TGF- $\beta 1$ signaling via down-regulating TGFBR2. miR-216a-5p may be a valuable target for anti-inflammation treatment in bronchopneumonia.

(c) 2021 Codon Publications. Published by Codon Publications.

\section{Introduction}

Bronchopneumonia is a frequent respiratory infection disease in children under 5 years of age. ${ }^{1}$ It is the most common hospitalization reason, and is one of the critical death causes for children. ${ }^{2}$ In China, the mortality rate is approximately 184 to1223/100,000 for children under 5 years. ${ }^{3,4}$ The common triggers causing bronchopneumonia are the infection of bacteria, viruses, mycoplasma, and other pathogens. Children are more susceptible to this infection because of the dysfunctional immune system. ${ }^{3,5}$ Increasing evidence revealed that inflammation is the primary response caused by bronchopneumonia infection, ${ }^{5,6}$ which indicates that anti-inflammation treatment may be an effective therapeutic strategy for bronchopneumonia. However, the potential mechanism of inflammation during bronchopneumonia remains unclear at present. Hence, it is imperative to investigate the detailed mechanism of inflammation underlying it.

MicroRNAs (miRNAs) are small noncoding RNAs with 18-25 nucleotides in length. ${ }^{7}$ In recent years, miRNAs have been proved to regulate many diseases including pneumonia, by repressing the expression of target genes.,9 For example, Zhang et al. ${ }^{9}$ revealed that miR-146b alleviated the inflammation injury in pediatric pneumonia by regulating MyD88/NF- $\mathrm{B}$ signaling pathway. In acute pneumonia, miR-370-3p modulated lipopolysaccharides (LPS)-induced WI-38 cell apoptosis and inflammation by binding to the toll-like receptor 4 (TLR4). ${ }^{6}$ miR-455-5p inhibited M1 macrophage polarization by binding to the suppressor of cytokine signaling 3 (SOCS3) in childhood pneumonia. ${ }^{10}$ In addition, miR-218 suppressed LPSinduced bronchial epithelial cell injury through targeting E2F Transcription Factor 2 (E2F2) in bronchopneumonia. ${ }^{2}$

As a member of the miRNAs family, miR-216a-5p is demonstrated to involve in some diseases. ${ }^{11,12}$ For instance, they attenuate oxidative stress caused by hydrogen peroxide via modulating the HMGB1/NF-kB pathway in human bronchial epithelial cell line 16HBE. ${ }^{11}$ miR-216a-5p inhibited tumor progression via binding to TPT1/mTORC1 in pancreatic cancer. ${ }^{12}$ Furthermore, accumulating evidence revealed that miR216a-5p modulated inflammation response in some diseases. ${ }^{13,14}$ Kong et al. ${ }^{13}$ reported that miR-216a attenuated LPS-induced inflammatory injury through modulating JAK2/STAT3 and $N F-\kappa B$ signaling pathways in acute lung injury. Peng et al. ${ }^{14}$ reported that miR-216a-5p-containing exosomes suppressed rTp17-caused inflammatory via binding to TLR4. However, the action of miR-216a-5p on bronchopneumonia is not elucidated at present. Given the regulatory effect of miR-216a-5p on inflammation response, it was inferred that miR-216a-5p might affect bronchopneumonia development. Hence, this study focuses on investigating the action of miR-216a-5p on bronchopneumonia and study the detailed mechanism underlying it.

\section{Materials and methods}

\section{Cell culture}

Human bronchial epithelial cell line BEAS-2B was acquired from American Type Culture Collection (ATCC, Manassas, VA) and maintained in Dulbecco's Modified Eagle medium with 10\% fetal bovine serum at $37^{\circ} \mathrm{C}$ with $5 \%$ carbon-di-oxide. Later, cells were stimulated with LPS of $1,5,10,20$, or $30 \mu \mathrm{M}$, respectively (Sigma, St. Louis, MO, USA) for 6, 12, 24, or 48 hours.

\section{Quantitative real-time polymerase chain reaction ( $q R T-P C R)$}

RNA of BEAS-2B cells were isolated using TRIzol (Sigma). Complementary DNA was generated through reverse transcription reaction using the Sensiscript II reverse transcriptase (Qiagen, Hilden, Germany), followed by a qPCR assay using SYBR Green PCR master mix (Qiagen, Hilden, Germany). The primers used in the study were: miR-216a-5p: F, 5'TGTCGCAAATCT CTGCAGG-3', R, 5'-CAGAGCAGGGTCCGAGG TA-3'; '15 Interleukin (IL)-1 $\beta$ :F, 5'-GGATATGGAGCAACAAGTGG-3', R, 5'-ATGTACCAGTTGGGGAACTG-3';16 IL-6: F, 5'-AAATTCGG TACATCCTCGACGG-3', R, 5'-GGAAGGTTCAGGTTGTTTTCTGC3';17 Tumor necrosis factor (TNF)- $\alpha$ : F, 5'-TAATGCTGATTT GGTGACCAGG-3', F, 5'-GTAGGGCGATTACAGTCACGG-3';18

Transforming growth factor-beta receptor 2 (TGFBR2): F, 5'-ACGGCGTTACAGTGTTTCTG-3'; R, 5'-GCACATACAAA 
CGGCCTATCTC-3'. ${ }^{19} \beta$-actin and U6 served as control genes for data normalization. Relative gene expression was analyzed by the $2^{-\Delta \Delta \mathrm{ct}}$ method. ${ }^{20}$

\section{Enzyme-linked immunosorbent assay (ELISA)}

The production of IL- $1 \beta$, IL- 6 , and TNF- $\alpha$ in BEAS-2B cells were determined using the corresponding human ELISA kit (R\&D Systems, Inc., Minneapolis, MN, USA) following instructions of the manufacturer.

\section{Luciferase assay}

Bioinformatics analysis for miR-216a-5p targets was conducted using Targetscan (http://www.targetscan.org/). The wild and mutant sequences of TGFBR2 targeting miR-216a-5p were fused into the pmirGLO vector (Promega, Madison, WI, USA). The cloned pmirGLO vector and miR-216a-5p mimic were transfected into BEAS-2B cells. Forty-eight hours post transfection, a dual-luciferase reporter assay system (Promega, Madison, WI, USA) was employed to measure luciferase activity.

\section{Cell transfection}

miR-216a-5p mimic and TGFBR2 overexpressed plasmid were acquired from Sangon Biotech (Sangon Biotech, Shanghai, China) and were transfected into BEAS-2B cells utilizing Lipofectamine 3000 (Thermo Scientific, Waltham, MA, USA) following the instructions of the manufacturer. Forty-eight hours post-transfection, the cells were exposed to $10 \mu \mathrm{M}$ LPS for 48 hours.

\section{Western blot}

Total protein extraction from BEAS-2B cells was conducted utilizing radioimmunoprecipitation assay buffer and quantified using a BCA kit (Sigma). Extracted proteins were subjected to sodium dodecyl sulfate-polyacrylamide gel electrophoresis and then transferred onto a polyvinylidene fluoride membrane. After blocking for nonspecific binding, the membrane was labeled with anti-TGFBR2 (1:2000; ab186838), TGF- $\beta 1$ (1:2000; ab92486), p-SMAD family member 2 (smad2; 1:2000; ab188334), smad2 (1:2000; ab228765), and $\beta$-actin (1:3000) antibodies (Abcam, Cambridge, MA,
UK) at $4^{\circ} \mathrm{C}$ for $12 \mathrm{~h}$. Then, they were further treated with goat anti-mouse IgG H\&L (Abcam, Cambridge, MA, UK) for 2 hours. $\beta$-actin was chosen as the control protein. The blots were studied using the ECL Plus Western Blotting Substrate (Thermo Fisher Scientific, Waltham, MA, USA).

\section{Statistical analysis}

Data were described as mean \pm standard deviation (SD), and data processing was conducted by SPSS Statistics 22.0 (SPSS, Chicago, IL, USA). One-way analysis of variance with the least significant difference post hoc test was applied for multiple group comparisons. $\mathrm{P}<0.05$ was the principle of statistical significance.

\section{Results}

\section{miR-216a-5p expression decreased in LPS-induced BEAS-2B cells}

To interpret the action of miR-216a-5p on the bronchopneumonia of children. BEAS-2B cells were first exposed to LPS to imitate the bronchopneumonia cell model. Later, miR-216a-5p expression in LPS-induced BEAS-2B was determined. miR-216a-5p was down-regulated in BEAS-2B cells after being stimulated with $1,5,10,20$, or $30 \mu \mathrm{M}$ LPS, respectively, for 48 hours ( $P<0.01$; Figure $1 A)$. Especially, miR-216a-5p presented the lowest expression in BEAS-2B cells stimulated by $10 \mu \mathrm{M}$ LPS (Figure 1A). Therefore, $10 \mu \mathrm{M}$ LPS was selected to induce BEAS-2B cells in the follow-up experiments. In addition, miR-216a-5p expression decreased in BEAS-2B cells after $10 \mu \mathrm{M}$ LPS treatment in a time-dependent manner $(P<0.01$; Figure $1 B)$. Therefore, miR-216a-5p was down-regulated in LPS-stimulated BEAS-2B cells.

\section{miR-216a-5p expression suppressed LPS-induced inflammation in BEAS-2B cells}

For better exploration of the role of miR-216a-5p in bronchopneumonia, miR-216a-5p mimic was introduced into BEAS-2B cells miR-216a-5p mimic complemented LPS-caused decrease of miR-216a-5p $(P<0.01$; Figure 2A). Moreover, LPS-induced upregulation of IL-1 $\beta, I L-6$, and TNF- $\alpha$ levels was reversed by the miR-216a-5p mimic ( $P<0.01$; Figure $2 B, C)$.
(A)

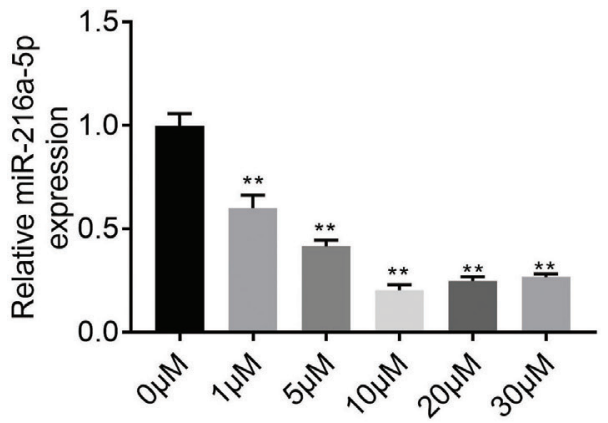

(B)

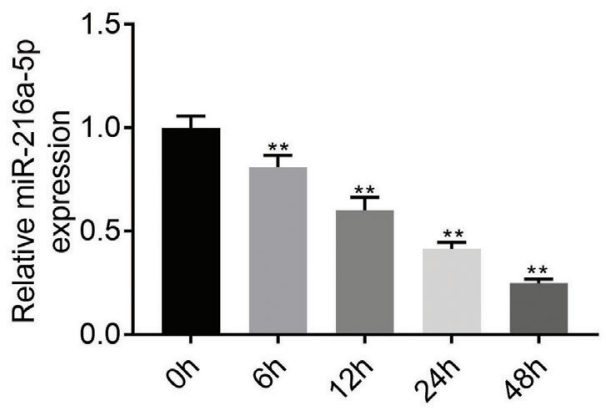

Figure 1 miR-216a-5p was down-regulated in LPS-induced BEAS-2B cells. (A) The expression of miR-216a-5p was detected using qRT-PCR in BEAS-2B cells treated with $0,1,5,10,20$, or $30 \mu \mathrm{M}$ LPS, respectively. The experiment was performed in triplicates. (B) The expression of miR-216a-5p was detected using qRT-PCR in BEAS-2B cells treated with $10 \mu \mathrm{M}$ LPS for $0,6,12,24$, or 48 hours, respectively. The experiment was performed in triplicates. ${ }^{*} \mathrm{P}<0.01$. 
(A)

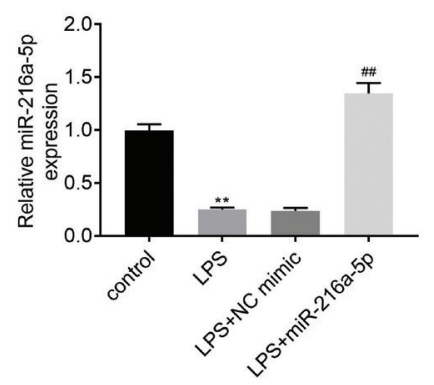

(C)

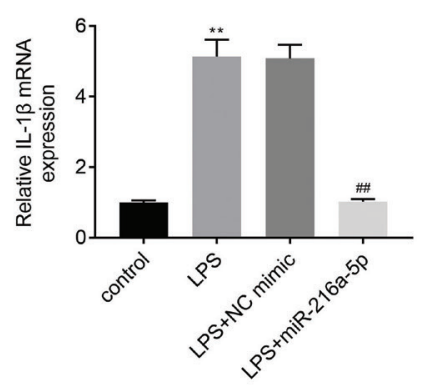

(B)
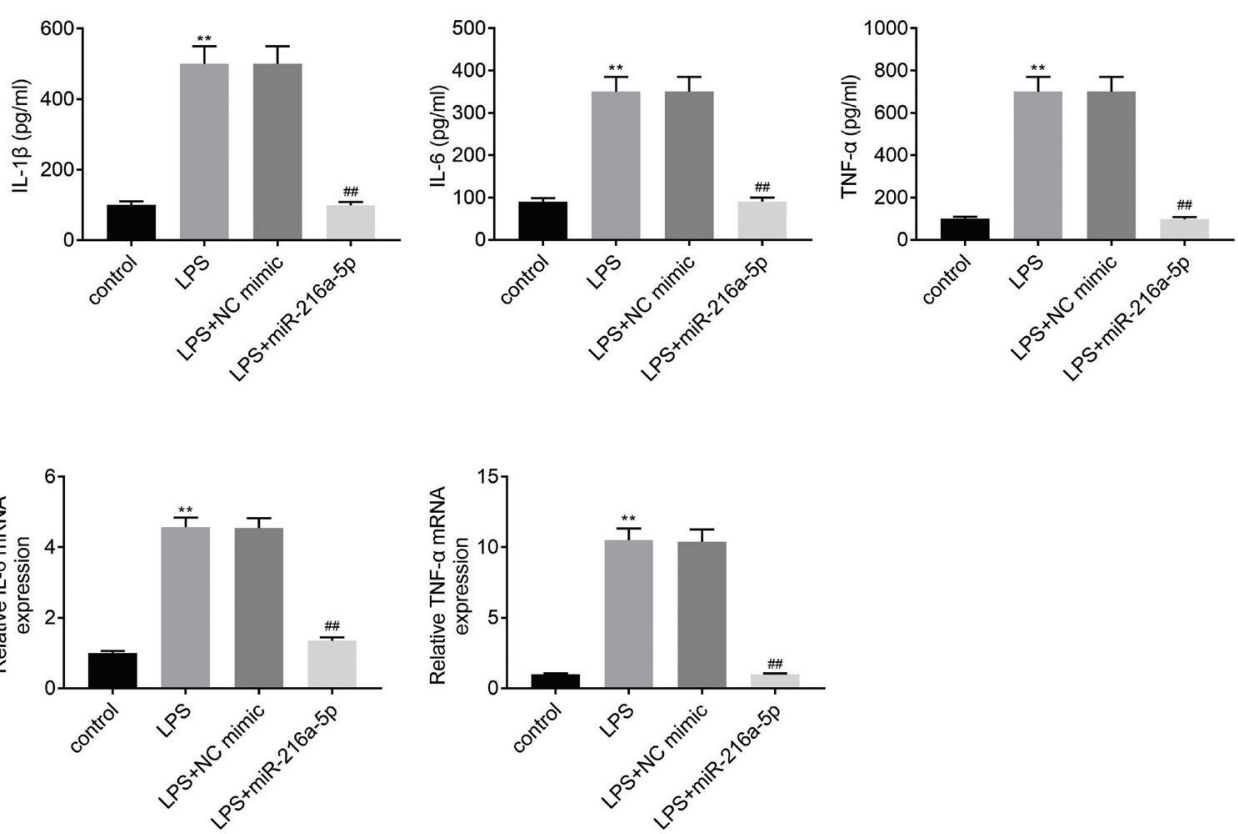

Figure 2 miR-216a-5p suppressed LPS-induced inflammation in BEAS-2B cells. (A) qRT-PCR was used to determine miR-216a-5p expression after BEAS-2B cells were transfected with miR-216a-5p mimic and treated with LPS. The experiment was performed in triplicates. (B) The levels of IL-1 $\beta$, IL-6, and TNF- $\alpha$ in BEAS-2B cells transfected with miR-216a-5p mimic and treated with LPS were determined using ELISA assay in triplicates. (C) The mRNA expression levels of IL-1 $\beta$, IL-6, and TNF- $\alpha$ in BEAS-2B cells transfected with miR-216a-5p mimic and treated with LPS were determined by qRT-PCRin triplicates. ${ }^{* *} \mathrm{P}<0.01$. \#P $<0.01$.

Thus, miR-216a-5p suppressed LPS-caused inflammation in BEAS-2B cells.

\section{TGFBR2 was a target of miR-216a-5p}

To investigate the mechanism of miR-216a-5p alleviating LPS-caused inflammation in BEAS-2B cells, the underlying targets of miR-216a-5p were screened by bioinformatics analysis. Using the Targetscan database, TGFBR2 was found to be a target of miR-216a-5p, and the potential target sequence of TGFBR2 and miR-216a-5p are listed in Figure 3A. Later, a luciferase assay was performed to verify the relationship between miR-216a-5p and TGFBR2. Results proved that miR-216a-5p mimic caused a noticeable reduction in the activity of wild 3'-UTR of TGFBR2 reporter but did not affect mutant 3'-UTR of TGFBR2 reporter $(\mathrm{P}<$ 0.01; Figure $3 B$ ). Besides, $q R T-P C R$ results revealed that LPS induced TGFBR2 mRNA expression but was inhibited by miR-216a-5p mimic ( $P<0.01$; Figure $3 C)$. Furthermore, Western blot results found that the promotional effect of LPS on the TGFBR2 protein level was remarkably abolished by miR-216a-5p mimic ( $P<0.01$, Figure $3 D$ ). In conclusion, TGFBR2 was a direct target of miR-216a-5p, and it negatively regulated TGFBR2 expression.

\section{miR-216a-5p inhibited the TGF- $\beta 1$ signaling pathway by modulating TGFBR2}

Previous studies reported that the TGF- $\beta 1$ signaling participated in pulmonary inflammation regulation. ${ }^{21,22}$
This study investigated if the TGF- $\beta 1$ signaling pathway was associated with the modulation of miR-216a-5p on LPS-caused inflammation. After BEAS-2B cells were introduced into miR-216a-5p mimic and TGFBR2 overexpression plasmid and treated with LPS, the protein levels of TGFBR2, TGF- $\beta 1$, and phosphorylation of smad2 were detected. Results revealed that miR-216a-5p mimic the restrained LPS-induced protein levels of TGFBR2 and TGF- $\beta 1$, which was seen to be reversed by overexpressed TGFBR2 ( $P<0.01$; Figure 4). In addition, LPS induced phosphorylation of smad2 was repressed by miR-216a-5p mimic and was observed to be reversed by overexpressed TGFBR2 later $(P<0.01 ;$ Figure 4). Hence, miR-216a-5p suppressed the TGF- $\beta 1$ signaling pathway through modulating TGFBR2.

\section{miR-216a-5p suppressed LPS-induced inflamma- tion in BEAS-2B cells via regulating TGFBR2}

To investigate the underlying mechanism of the regulation of miR-216a-5p on LPS-induced inflammation. The BEAS-2B cells were cotransfected with miR-216a-5p mimic and TGFBR2 overexpression plasmid and treated with LPS. The expression levels of IL-1 $\beta$, IL- 6 , and TNF- $\alpha$ were then determined. Results showed that miR-216a-5p mimic suppressed LPS-induced mRNA levels of IL-1 $\beta$, IL-6, and TNF- $\alpha$, which were seen to be restored by overexpressed TGFBR2 $(P<0.01$; Figure 5). Therefore, these findings suggested that miR-216a-5p suppressed LPS-induced inflammation in BEAS-2B cells via TGFBR2 regulation. 
(A)

$\begin{array}{ll}\text { TGFBR2-wt } & \text { 5'... CACUUCCUCAGGAAAUGAGAUUG ...3' } \\ \text { hsa-miR-216a-5p } & \text { 3' AGUGUCAACGGUCGACUCUAAU } \\ \text { TGFBR2-mut } & \text { 5'... CACUUCCUCAGGAAAACUCUAAG ...3' }\end{array}$

(B)

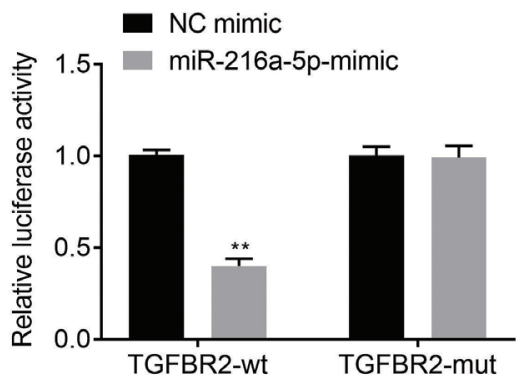

(C)

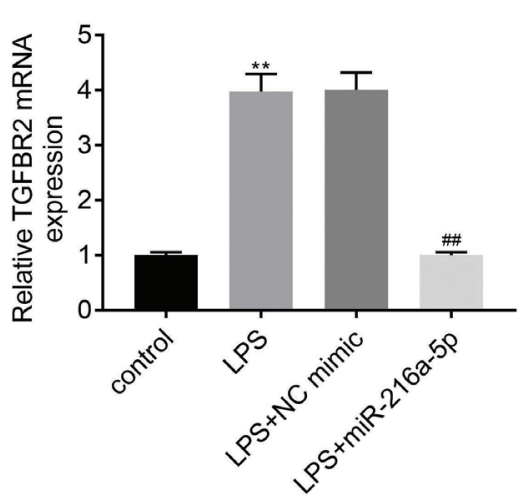

(D)

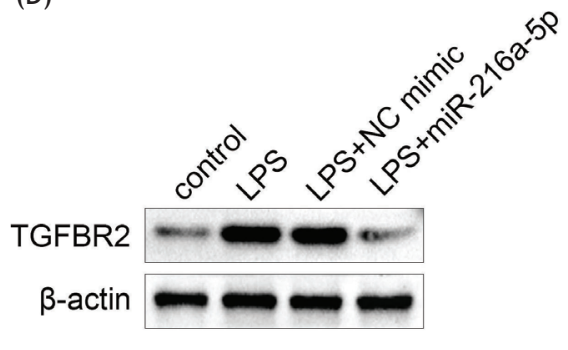

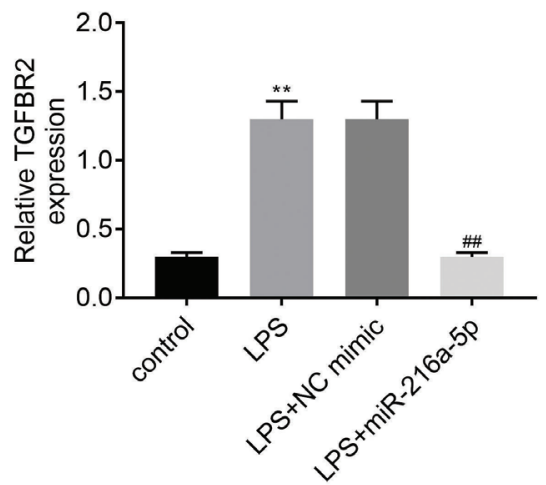

Figure 3 TGFBR2 was a direct target of miR-216a-5p. (A) The putative binding sequences of miR-216a-5p and TGFBR2. (B) The luciferase reporter assay was conducted to verify the relationship between miR-216a-5p and TGFBR2. The experiment was performed four times. (C) The mRNA expression of TGFBR2 in BEAS-2B cells transfected with miR-216a-5p mimic and treated with LPS were determined by qRT-PCR. The experiment was performed four times (D)The protein level of TGFBR2 in BEAS-2B cells transfected with miR-216a-5p mimic and treated with LPS were determined by the Western blot. The experiment was performed four times. ${ }^{* *} P<0.01$. ${ }^{\# \# P}<0.01$.

\section{Discussion}

As a common respiratory infection disease, bronchopneumonia is the leading cause of hospitalization in children. ${ }^{1,2}$ At present, anti-inflammation treatment may be an effective therapeutic strategy for bronchopneumonia because it causes inflammation response. ${ }^{5,6}$ However, the potential mechanism of inflammation response in bronchopneumonia has not been fully elucidated. miR-216a-5p is proved to modulate inflammation response, ${ }^{14}$ whereas its effect on bronchopneumonia was unclear. Therefore, this study explored the action of miR-216a-5p on bronchopneumonia in this study.

Studies revealed that LPS could cause endogenous bioactive factors production, thereby triggering inflammation response. ${ }^{5,23}$ Therefore, LPS was used to treat BEAS-2B cells to simulate bronchopneumonia in vitro. miR-216a-5p expression was determined in BEAS-2B cells stimulated by LPS. The study outcomes revealed that miR-216a-5p declined in LPS-treated BEAS-2B cells, which was in line with the Kong et al study. ${ }^{13}$ They showed that miR-216a-5p expression decreased in acute lung injury caused by LPS. ${ }^{13}$ The aberrant expression of miR-216a-5p in BEAS-2B cells exposed to LPS indicated that it might exert a regulation role in bronchopneumonia. To investigate the action of miR-216a-5p on bronchopneumonia. The effect of the miR216a-5p expression on inflammatory cytokines secretion was explored. Results revealed that overexpressed miR216a-5p suppressed the secretion of IL-1 $1 \beta$, IL-6, and TNF- $\alpha$ caused by LPS in BEAS-2B cells. The regulatory effect of miR-216a-5p on inflammation response has already been reported in other diseases.14,24 Pent et al. ${ }^{14}$ revealed that decreased miR-216a-5p in syphilis patients was negatively associated to the levels of IL-1 $\beta$, IL- 6 , and TNF- $\alpha$, and miR-216a-5p-containing exosomes alleviated rTp17-caused inflammation. Similarly, Wang et al. ${ }^{24}$ revealed that overexpressed miR-216a-5p inhibited the levels of IL-6, TNF- $\alpha$, and IL-1 $\beta$ in neuropathic pain caused by chronic constriction injury. Overall, these findings indicated that miR-216a-5p suppressed LPS-caused inflammation in BEAS-2B cells.

Accumulating evidence demonstrated that miRNAs usually played a regulatory role in diseases by binding to the target gene. ${ }^{25}$ Therefore, to elucidate the potential mechanism of miR-216a-5p on LPS-caused inflammation in BEAS-2B cells, the targets of miR-216a-5p were searched. The findings revealed that TGFBR2 was the target of miR216a-5p, and it negatively modulated the TGFBR2 expression. This target relation of TGFBR2 and miR-216a-5p has not been reported. TGFBR2 is a transmembrane serine/ threonine kinase, and it is the critical member of the TGF- $\beta 1$ signaling pathway. ${ }^{26,27} \mathrm{~A}$ previous study proved that suppressing TGFBR2 inhibited TGF- $\beta 1$ signal transduction, thereby alleviating inflammation response and apoptosis in the model of bronchopulmonary dysplasia. ${ }^{21}$ In addition, 

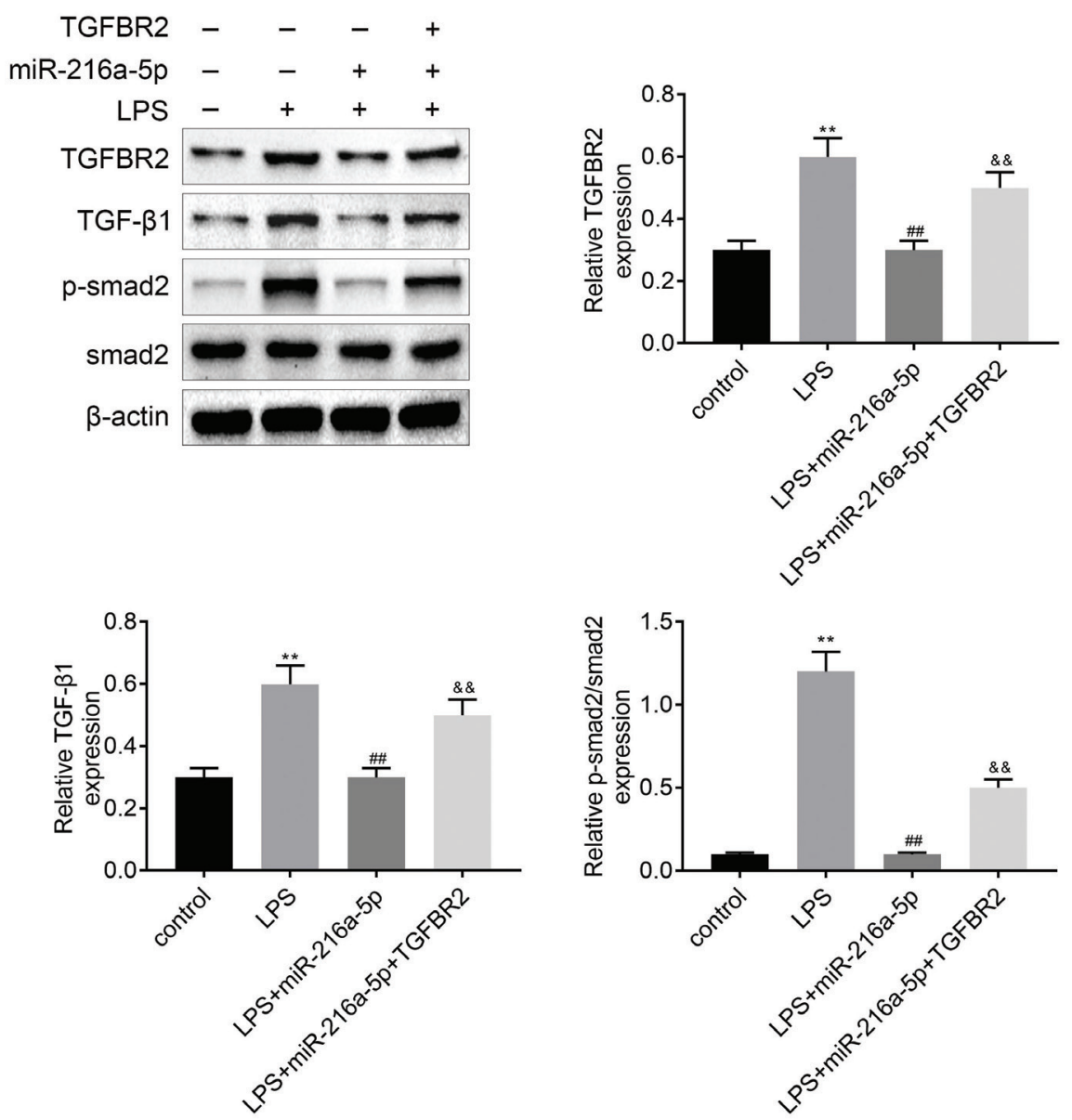

Figure 4 miR-216a-5p suppressed the TGF- $\beta 1$ signaling pathway through modulating TGFBR2. Western blot was used to determine the protein levels of TGFBR2, TGF- $\beta 1$, and phosphorylation of smad2 after BEAS-2B cells were transfected with miR-216a-5p mimic, TGFBR2 overexpression plasmid, and treated with LPS. The experiment was performed three times. ${ }^{* *} \mathrm{P}<0.01$. ${ }^{\# \# P}<0.01$.
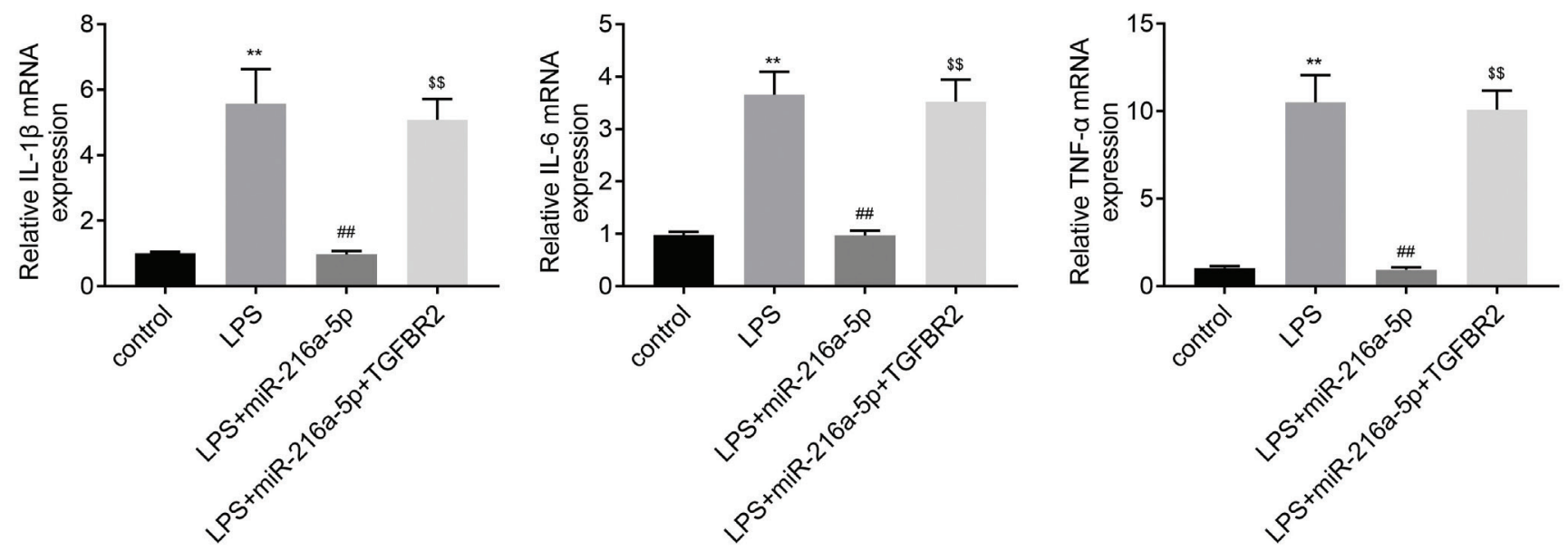

Figure 5 miR-216a-5p suppressed LPS-induced inflammation in BEAS-2B cells via regulating TGFBR2. qRT-PCR was used to determine the mRNA levels of IL-1 $\beta$, IL-6, and TNF- $\alpha$ after BEAS-2B cells were transfected with miR-216a-5p mimic, TGFBR2

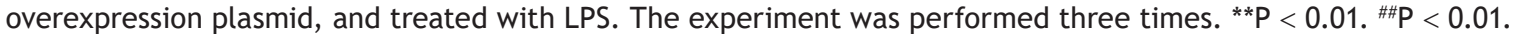

other researches showed that the TGF- $\beta 1$ signaling participated in the regulation of pulmonary inflammation, ${ }^{22}$ and its expression was elevated in the bronchoalveolar lavage fluid of patients with idiopathic eosinophilic pneumonia. ${ }^{28}$ Thus, this study inferred that miR-216a-5p might also regulate TGF- $\beta 1$ signaling in bronchopneumonia. The outcomes showed that overexpressed miR-216a-5p restrained LPS-induced protein levels of TGFBR2, TGF- $\beta 1$, and phosphorylation of smad2, which was seen to be reversed by overexpressed TGFBR2. In other words, miR-216a-5p 


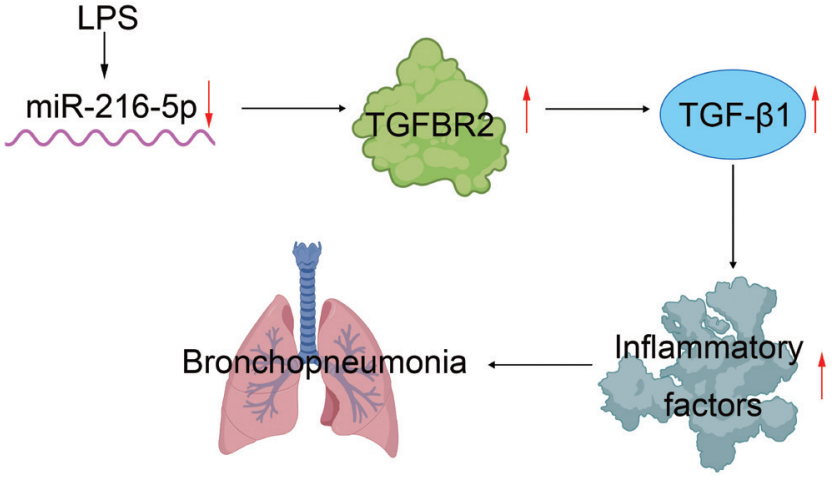

Figure 6 Proposed molecular mechanisms of miR-216a-5p on inflammation in bronchopneumonia. LPS decreased miR216a-5p expression, and the decreased miR-216a-5p elevated TGFBR2 expression, thereby activating the TGF- $\beta 1$ signaling pathway, inducing inflammatory factor production and consequently bronchopneumonia.

suppressed the TGF- $\beta 1$ signaling pathway through modulating TGFBR2. Furthermore, the investigation of the underlying mechanism of miR-216a-5p on LPS-induced inflammation in the human bronchial epithelial cell revealed that miR-216a-5p suppressed LPS-induced inflammation in BEAS-2B cells via regulating TGFBR2. Thus, this study hypothesized that the following regulatory pathway in bronchopneumonia: LPS stimulation induced a decrease in miR-216a-5p expression, and this miR-216a-5p elevated TGFBR2 expression, thereby activating the TGF- $\beta 1$ signaling pathway, which induced inflammatory factor production and consequently bronchopneumonia (Figure 6). Additionally, ectopic expression of miR-216a-5p alleviated LPS-induced inflammation in the human bronchial epithelial cell by inhibiting TGF- $\beta 1$ signaling via down-regulating TGFBR2.

\section{Conclusion}

miR-216a-5p suppressed LPS-induced inflammation in BEAS-2B cells by the inhibition of TGF $\beta 1$ signaling via down-regulating TGFBR2. miR-216a-5p may be a valuable target for anti-inflammation treatment in bronchopneumonia.

\section{Conflict of interest}

No potential conflict of interest was reported by the author.

\section{References}

1. Lindström L, Tauni FA, Vargmar K. Bronchopneumonia in Swedish lambs: A study of pathological changes and bacteriological agents. Acta Veterinaria Scandinavica. 2018;60(1):1-8. https://doi.org/10.1186/s13028-018-0409-1

2. Gong M, Mao Z, Chen J, Ye X, Zhou X. microRNA-218 inhibits lipopolysaccharide-induced bronchial epithelial cell injury by targeting E2F2. J Biomater Tissue Eng. 2020;10(8):1161-1169. https://doi.org/10.1166/jbt.2020.2377
3. You C, Ran G, Wu X, Wang Y, Tian H, Fan J, et al. High immunoglobulin $\mathrm{E}$ level is associated with increased readmission in children with bronchopneumonia. Ther Adv Respir Dis. 2019;13:1753466619879832. https://doi. org/10.1177/1753466619879832

4. Guan X, Silk BJ, Li W, Fleischauer AT, Xing X, Jiang X. Pneumonia incidence and mortality in Mainland China: Systematic review of Chinese and English literature, 1985-2008. PLoS One. 2010;5(7):e11721. https://doi.org/10.1371/journal.pone.0011721

5. Liu X, Meng J. Luteolin alleviates LPS-induced bronchopneumonia injury in vitro and in vivo by down-regulating microRNA132 expression. Biomed Pharmacother. 2018;106:1641-1649. https://doi.org/10.1016/j.biopha.2018.07.094

6. Zhang Y, Zhu Y, Gao G, Zhou Z. Knockdown XIST alleviates LPS-induced WI-38 cell apoptosis and inflammation injury via targeting miR-370-3p/TLR4 in acute pneumonia. Cell Biochem Funct. 2019;37(5):348-58. https://doi.org/10.1002/cbf.3392

7. Vishnoi A, Rani S. MiRNA biogenesis and regulation of diseases: An overview. Methods Mol Bio. 2017;1509:1-10.https:// doi.org/10.1007/978-1-4939-6524-3_1

8. Reddy KB. MicroRNA (miRNA) in cancer. Cancer Cell Int. 2015;15(1):1-6. https://doi.org/10.1186/s12935-015-0185-1

9. Zhang L, Dong L, Tang Y, Li M, Zhang M. MiR-146b protects against the inflammation injury in pediatric pneumonia through MyD88/NF-KB signaling pathway. J Infect Dis. 2020;52(1):23-32. https://doi.org/10.1080/23744235.2019.1671987

10. Chi X, Ding B, Zhang L, Zhang J, Wang J, Zhang W. IncRNA GAS5 promotes M1 macrophage polarization via miR-455-5p/ SOCS3 pathway in childhood pneumonia. J Cell Physiol. 2019;234(8):13242-13251. https://doi.org/10.1002/jcp.27996

11. Chaoyang $Y$, Qingfeng $B$, Jinxing F. MiR-216a-5p protects 16HBE cells from $\mathrm{H} 2 \mathrm{O} 2$-induced oxidative stress through targeting HMGB1/NF-kB pathway. Biochem Biophys Res Commun. 2019;508(2):416-420. https://doi.org/10.1016/j. bbrc.2018.11.060

12. Zhang J, Gao S, Zhang Y, Yi H, Xu M, Xu J, et al. MiR-216a-5p inhibits tumorigenesis in pancreatic cancer by targeting TPT1/mTORC1 and is mediated by LINC01133. Int J Biol Sci. 2020;16(14):2612. https://doi.org/10.7150/ijbs.46822

13. Kong $F$, Sun $Y$, Song $W$, Zhou $Y$, Zhu S. MiR-216a alleviates LPS-induced acute lung injury via regulating JAK2/STAT3 and NF- $\kappa B$ signaling. Hum Cell. 2020;33(1):67-78. https://doi. org/10.1007/s13577-019-00289-7

14. Peng R-R, Shang S-X, Zhao L-S, Long F-Q. MiR-216a-5pcontaining exosomes suppress $\mathrm{rTp} 17$-induced inflammatory response by targeting TLR4. Biosci Rep. 2019;39(8): BSR20190686. https://doi.org/10.1042/BSR20190686

15. Sun $Y$, Hu B, Wang $Y$, Li Z, Wu J, Yang $Y$, et al. miR-216a-5p inhibits malignant progression in small cell lung cancer: Involvement of the Bcl-2 family proteins. Cancer Manag Res. 2018;10:4735. https://doi.org/10.2147/CMAR.S178380

16. Le Jan S, Muller C, Plee J, Durlach A, Bernard P, Antonicelli F. IL-23/IL-17 axis activates IL-1 $\beta$-associated inflammasome in macrophages and generates an auto-inflammatory response in a subgroup of patients with bullous pemphigoid. Front Immunol. 2019;10:1972. https://doi.org/10.3389/fimmu.2019.01972

17. Yu T, Xu Y-Y, Zhang Y-Y, Li K-Y, Shao Y, Liu G. Plumbagin suppresses the human large cell lung cancer cell lines by inhibiting IL-6/STAT3 signaling in vitro. Int Immunopharmacol. 2018;55:290-296. https://doi.org/10.1016/j.intimp.2017.12.021

18. Han R, Zhang F, Wan C, Liu L, Zhong Q, Ding W. Effect of perfluorooctane sulphonate-induced Kupffer cell activation on hepatocyte proliferation through the NF- $\mathrm{B} / \mathrm{TNF}-\alpha /$ IL-6-dependent pathway. Chemosphere. 2018;200:283-294. https://doi.org/10.1016/j.chemosphere.2018.02.137

19. Fan Y, Gao Y, Rao J, Zhang F, Wang K, Zhang C. YAP-1 promotes tregs differentiation in hepatocellular carcinoma by enhancing TGFBR2 transcription. Cell Physiol Biochem. 2017;41(3):1189-1198. https://doi.org/10.1159/000464380 
20. Livak KJ, Schmittgen TD. Analysis of relative gene expression data using real-time quantitative PCR and the 2(-Delta Delta C(T)) Method. Methods. 2001;25(4):402-408. https://doi. org/10.1006/meth.2001.1262

21. Sureshbabu A, Syed MA, Boddupalli CS, Dhodapkar MV, Homer RJ, Minoo P, et al. Conditional overexpression of TGF $\beta 1$ promotes pulmonary inflammation, apoptosis and mortality via TGF $\beta$ R2 in the developing mouse lung. Respir Res. 2015;16(1):1-12. https://doi.org/10.1186/ s12931-014-0162-6

22. Ganeshan K, Johnston LK, Bryce PJ. TGF- $\beta 1$ limits the onset of innate lung inflammation by promoting mast cell-derived IL-6. J Immunol. 2013;190(11):5731-5738. https://doi.org/10.4049/ jimmunol.1203362

23. Lykhmus O, Mishra N, Koval L, Kalashnyk O, Gergalova G, Uspenska $\mathrm{K}$, et al. Molecular mechanisms regulating LPSinduced inflammation in the brain. Front Mol Neurosci. 2016;9:19. https://doi.org/10.3389/fnmol.2016.00019

24. Wang W, Li R. MiR-216a-5p alleviates chronic constriction injury-induced neuropathic pain in rats by targeting KDM3A and inactivating Wnt/ $\beta$-catenin signaling pathway. Neurosci Res. 2020; S0168-0102(20)30421-1. https://doi.org/10.1016/j. neures.2020.08.001

25. Pu M, Chen J, Tao Z, Miao L, Qi X, Wang Y, et al. Regulatory network of miRNA on its target: Coordination between transcriptional and post-transcriptional regulation of gene expression. Cell Mol Life Sci. 2019;76(3):441-451. https://doi. org/10.1007/s00018-018-2940-7

26. Hart PJ, Deep S, Taylor AB, Shu Z, Hinck CS, Hinck AP. Crystal structure of the human TbetaR2 ectodomain--TGF-beta3 complex. Nat Struc Biol. 2002;9(3):203-208. https://doi. org/10.1038/nsb766

27. Massagué J, Seoane J, Wotton D. Smad transcription factors. Genes Dev. 2005;19(23):2783-810. https://doi.org/10.1101/ gad. 1350705

28. Katoh S, Matsumoto N, Tanaka H, Yasokawa N, Kittaka M, Kurose $\mathrm{K}$, et al. Elevated levels of periostin and TGF- $\beta 1$ in the bronchoalveolar lavage fluid of patients with idiopathic eosinophilic pneumonia. Asian Pac J Allergy Immunol.. 2020;38(3):208-213. https://doi.org/10.12932/AP-111018-0414 